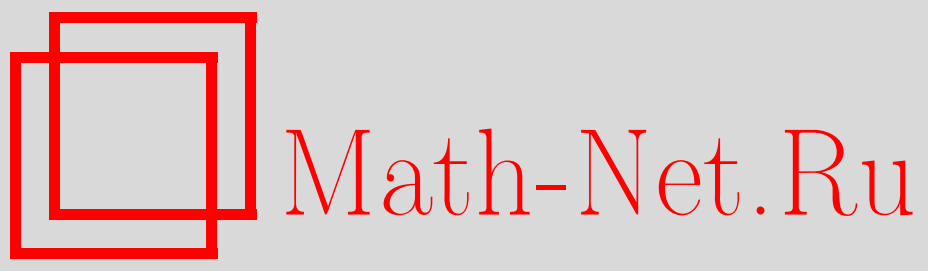

С. Буаррудж, П. Я. Грозман, Д. А. Лейтес, Новые простые модулярные супералгебры Ли как обобщенные продолжения, Функи. анализ и его прил., 2008, том 42, выпуск 3, 1-9

DOI: https://doi.org/10.4213/faa2924

Использование Общероссийского математического портала MathNet.Ru подразумевает, что вы прочитали и согласны с пользовательским соглашением

http://www . mathnet.ru/rus/agreement

Параметры загрузки:

IP : 54.209 .52 .79

26 апреля 2023 г., $17: 33: 48$

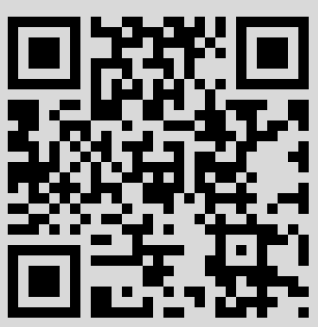


Функиионалъный анализ и его приложения

2008, т. 42, вып. 3, с. 1-9

УДК 512.554 .38

\title{
Новые простые модулярные супералгебры Ли как обобщенные продолжения*
}

\author{
(C) 2008. С. БУАРрУДЖ, П. Я. ГрОзМАН, Д. А. ЛЕЙтЕС
}

\section{§1. Постановка задачи}

Всюду в этой работе $\mathbb{K}$ - алгебраически замкнутое поле характеристики $p>2$.

Классические работы С. Ли, В. Киллинга и Э. Картана завершили классификацию над полем $\mathbb{C}$ следующих типов простых алгебр Ли:

всех конечномерных и некоторых бесконечномерных

( а именно, состолщих из полиномиальных векторных полей).

В 1930-х годах топологи стали рассматривать алгебры Ли и супералгебры Ли над полями характеристики $p>0$, называемые также модулярными (супер)алгебрами Ли. Простые модулярные алгебры Ли (над конечными полями $\mathbb{K}$ ) привлекли внимание как побочный продукт классификации конечных простых групп. Супералгебры Ли, даже простые и даже над полями $\mathbb{C}$ или $\mathbb{R}$, не особенно интересовали математиков до 1970-х годов, когда физики обнаружили их (выдающуюся) полезность, однако модулярные супералгебры Ли практически не интересовали исследователей до недавнего времени.

С 1930-х годов математики открывали все новые и новые примеры простых модулярных алгебр Ли, пока Кострикин и Шафаревич [15] не сформулировали для $p>7$ гипотезу (КШ-гипотезу), охватившую все известные к этому времени примеры и верную, как оказалось, не только при $p>7$, но и при $p=7$. Чтобы сформулировать (обобщенную) КШ-гипотезу и ее супер-аналог, напомним необходимые понятия и методы. Например, все простые конечномерные алгебры Ли над $\mathbb{C}$ имеют вид $\mathfrak{g}(A)$, где $A$ - матрица, называемая матрицей Картана алгебры Ли $\mathfrak{g}(A)$.

1.1. Что такое $\mathfrak{g}(\boldsymbol{A})$. (Супер)алгебры Ли вида $\mathfrak{g}(A)$ (иногда их называют контраградиентными наряду с некоторыми другими алгебрами, у которых матрицы Картана нет) определяются следующим образом. Пусть $A=\left(A_{i j}\right)-$ произвольная матрица размера $n \times n$ ранга $l$ с элементами из поля $\mathbb{K}$. Фиксируем векторное пространство $\mathfrak{h}$ размерности $2 n-l$ и его дуальное $\mathfrak{h}^{*}$ и выберем $n$ линейно независимых векторов $h_{i} \in \mathfrak{h}$ и $n$ линейно независимых векторов $\alpha_{j} \in \mathfrak{h}^{*}$ так, что $\alpha_{i}\left(h_{j}\right)=A_{i j}$.

Пусть $I=\left\{i_{1}, \ldots, i_{n}\right\} \subset(\mathbb{Z} / 2 \mathbb{Z})^{n} ;$ рассмотрим свободную супералгебру Ли $\tilde{\mathfrak{g}}(A, I)$, порожденную генераторами $e_{1}^{ \pm}, \ldots, e_{n}^{ \pm}$, для которых $p\left(e_{j}^{ \pm}\right)=i_{j}$, где $p-$

* Мы благодарны А. В. Лебедеву и И. М. Щепочкиной за помощь; Д. Л. благодарит институт MPIMiS, Лейпциг, где эта статья опубликована как препринт 146/2006, за финансовую подержку. 
четность в $\tilde{\mathfrak{g}}(A, I)$, и пространством $\mathfrak{h}$, с соотношениями

$$
\begin{gathered}
{\left[e_{i}^{+}, e_{j}^{-}\right]=\delta_{i j} h_{i} \text { для всех } i, j, \quad[\mathfrak{h}, \mathfrak{h}]=0,} \\
{\left[h, e_{j}^{ \pm}\right]= \pm \alpha_{j}(h) e_{j}^{ \pm} \text {всех } h \in \mathfrak{h} \text { и для всех } j}
\end{gathered}
$$

(здесь в каждом равенстве индексы \pm либо все равны + , либо все равны - ). Пусть $\tilde{\mathfrak{g}}_{ \pm}$- подалгебры в алгебре $\tilde{\mathfrak{g}}(A, I)$, порожденные всеми элементами $e_{i}^{ \pm}$.

Предложение. Среди идеалов в $\tilde{\mathfrak{g}}(A, I)$, пересекающихся $c \mathfrak{h}$ по нулю, имеется единственный максимальный идеал $\mathfrak{r}$, и он равен прямой сумме идеалов $\mathfrak{r} \cap \tilde{\mathfrak{g}}_{+} u \mathfrak{r} \cap \tilde{\mathfrak{g}}_{-}$.

Это утверждение хорошо известно над $\mathbb{C}$ для конечномерных и некоторых бесконечномерных алгебр Ли [14]; явное описание идеала $\mathfrak{r}$ для простых супералгебр Ли тех же типов над $\mathbb{C}$ см. в [12]; модулярный случай см. в [2], [3], [17].

Замена $A \mapsto \tilde{A}:=\operatorname{diag}\left(\lambda_{1}, \ldots, \lambda_{n}\right) \cdot A$, где $\lambda_{1}, \ldots, \lambda_{n} \in \mathbb{K} \backslash\{0\}$, сохраняет (супер)алгебру Ли $\tilde{\mathfrak{g}}(A, I)$. Мы будем называть матрицу Картана нормализованной, если она содержит на диагонали только 0,1 или 2 . Если $A_{j j}=2$, то $p\left(e_{j}^{ \pm}\right)=\overline{0}$, а чтобы отличать строки с $i_{j}=\overline{0}$ от строк с $i_{j}=\overline{1}$, мы будем писать $A_{j j}=\overline{0}$ или $\overline{1}$ (вместо $A_{j j}=0$ или 1 ), если $i_{j}=\overline{0}$. Для нормализованных матриц Картана, которые только и будут рассматриваться, нет нужды указывать $I$.

Подалгебра $\mathfrak{h}$ действует диагонально на $\tilde{\mathfrak{g}}(A)$, и ненулевые веса (супер)алгебры $\tilde{\mathfrak{g}}(A)$ относительно $\mathfrak{h}$ называются корнями. В модулярном случае удобнее использовать другое определение: (супер)алгебра $\tilde{\mathfrak{g}}(A)$ имеет $\mathbb{Z}^{n}$-градуировку, в которой $\operatorname{deg} e_{i}^{ \pm}=(0, \ldots, 0, \pm 1,0, \ldots, 0)$, где \pm 1 стоит на $i$-м месте (в случае $\mathbb{C}$ эта градуировка эквивалентна весовой), и мы будем называть корнями ненулевые степени этой градуировки, которым соответствуют ненулевые пространства. Для подмножества $B=\left\{\sigma_{1}, \ldots, \sigma_{n}\right\}$ корневой системы $R$ положим

$$
R_{B}^{ \pm}=\left\{\alpha \in R \mid \alpha= \pm \sum n_{i} \sigma_{i}, n_{i} \in \mathbb{Z}_{+}=\{0,1,2, \ldots\}\right\} .
$$

Множество $B$ называется системой простых корней корневой системы $R$ (или (супер)алгебры $\tilde{\mathfrak{g}}(A))$, если $\sigma_{1}, \ldots, \sigma_{n}$ линейно независимы и $R=R_{B}^{-} \coprod R_{B}^{+}$.

Положим $\mathfrak{g}(A)=\tilde{\mathfrak{g}}(A) / \mathfrak{r}$. Заметим, что модулярные (супер)алгебры Ли $\mathfrak{g}(A)$ могут не быть простыми, даже если выполняются условия, достаточные для простоты над $\mathbb{C}$.

Предупреждение. Если $m-n \equiv 0(\bmod p)$ (в частности, если $m=n$ над $\mathbb{C})$, то ни алгебра $\mathfrak{s l}(m \mid n)$, ни ее простой фактор ${ }^{1)} \mathfrak{p s l}(m \mid n)$ не имеют матрицы Картана - она есть у алгебры $\mathfrak{g l}(m \mid n)$.

1.2. Алгебры Ли векторных полей. Выбрав $\mathbb{Z}$-базис в алгебре многочленов от $m$ переменных $\mathbb{C}[x]$, состоящий из разделенных степеней

$$
u^{(r)}:=\prod u_{i}^{\left(r_{i}\right)}, \quad \text { где } u_{i}^{\left(r_{i}\right)}:=\frac{x_{i}^{r_{i}}}{r_{i} !},
$$

получим следующее семейство аналогов алгебры многочленов:

$$
\mathscr{O}(m ; \underline{N}):=\mathbb{K}[u ; \underline{N}]:=\operatorname{Span}_{\mathbb{K}}\left(u^{(r)} \mid r_{i}<p^{N_{i}} \text { для обрезающего вектора } \underline{N}\right),
$$

1) Если супералгебра Ли $\mathfrak{g} \subset \mathfrak{g r}(V)$ содержит пространство скалярных матриц $\mathfrak{s}=\mathbb{K} 1_{m \mid n}$, то положим $\mathfrak{p g}=\mathfrak{g} / \mathfrak{s}$ (проективизация). 
где $u=\left(u_{1}, \ldots, u_{m}\right), r=\left(r_{1}, \ldots, r_{m}\right), \underline{N}=\left(N_{1}, \ldots, N_{m}\right)$. Заметим, что из этих многочисленных алгебр разделенных степеней только одна порождается заявленными переменными - а именно та, для которой $N_{i}=1$ для всех $i$. В противном случае в список образующих к $u_{i}$ нужно добавить $u_{i}^{\left(p^{k_{i}}\right)}$ для всех $i$ и всех $k_{i}$, таких, что $1<k_{i}<N_{i}$. Поскольку любое дифференцирование алгебры определяется своими значениями на образующих, то, как мы видим, алгебра Ли $\mathfrak{d e r}(\mathscr{O}(m ; \underline{N}))$ всех дифференцирований алгебры $\mathscr{O}(m ; \underline{N})$ имеет больше чем $m$ функциональных параметров (коэффициентов при аналогах частных производных), если $N_{i} \neq 1$ хотя бы для одного $i$. Определим специальные частные производные, положив

$$
\partial_{i}\left(u_{j}^{(k)}\right)=\delta_{i j} u_{j}^{(k-1)} \text { для всех } k<p^{N_{j}} .
$$

Алгебра Ли всех дифференцирований $\mathfrak{d e r}(\mathscr{O}(m ; \underline{N}))$ оказывается менее интересной, чем ее подалгебра специальных дифференцирований, обозначаемая через $\mathfrak{v e c t}(m ; \underline{N})$, или $W(m ; \underline{N})$, или $\mathfrak{s d e r} \mathbb{K}[u ; \underline{N}]=\operatorname{Span}_{\mathbb{K}}\left(u^{(r)} \partial_{k} \mid r_{i}<p^{N_{i}}\right.$ при $\left.i \leqslant m\right)$

и называемая общей векторной алгеброй. Супералгебра Ли специальных дифференцирований определяется очевидным образом и обозначается через $\operatorname{vect}(m ; \underline{N} \mid n)$.

1.3. КШ-метод. Напомним, что (супер)алгебра Ли g называется ограниченной, если $\left(\operatorname{ad}_{X}\right)^{p}$ является внутренним дифференцированием для любого $X \in \mathfrak{g}$. Пусть $\mathfrak{g}^{(0)}:=\mathfrak{g}, \mathfrak{g}^{(i+1)}:=\left[\mathfrak{g}^{(i)}, \mathfrak{g}^{(i)}\right]$ - производные (супер)алгебры Ли $\mathfrak{g}$.

КШ-гипотеза. При $р>5$ простые ограниченные модулярные конечномерные алгебры Ли строятся одним из двух способов:

1) Для простой конечномерной алгебры Ли вида $\mathfrak{g}(A)$ над $\mathbb{C}$ возъмем матрииу Картана $A$, нормализованную таким образом, что $A_{i i}=2$ для всех $i$; въберем иелочисленный базис Шевалле (для нормализованной матрищы Картана такой базис - единственный с точностъю до знаков); символом $\mathfrak{g}_{\mathbb{Z}}$ обозначим алгебру Ли над $\mathbb{Z}$, порожденную базисом Шевалле, и положим $\mathfrak{g}_{\mathbb{K}}:=\mathfrak{g}_{\mathbb{Z}} \otimes_{\mathbb{Z}} \mathbb{K}$.

2) Для простой бесконечномерной алгебры Ли векторных полей g над $\mathbb{C}$ (возможно, сохраняющей тензор (борму объема, симплектическую или контактную форму)) возъмем в качестве $\mathfrak{g}_{\mathbb{K}}$ ее аналог, состояший из специалъных дифферениирований алгебры $\mathscr{O}(m ; \underline{N}=(1, \ldots, 1))$, сохраняющих модулярный аналог того эсе тензора.

Алгебры $\mathfrak{g}_{\mathbb{K}}$ просты (иногда с точностью до иентра или взятия первой или второй производной) и ограничены.

Обобщенная КШ-гипотеза. Описанные выше (в n. 2)) алгебры Ли $\mathfrak{g}_{\mathbb{K}}$, но с любым $\underline{N}$, и их дебормации ${ }^{1)}$ дают все простые конечномерные алгебры Ли над алгебраически замкнутыми полями, если $p>5$. При $p=5 \kappa$ списку нужно также добавить примерь Меликяна (описанные ниже).

1) Хотя в [18] даны основания считать, что стандартное определение универсальной обертывающей алгебры нуждается в изменении в модулярном случае, а вместе с ним и определение (ко)гомологий, для ограниченных (супер)алгебр Ли вида $\mathfrak{g}(A)$ и их «родственников» инфинитезимальные деформации можно описывать по-старому в терминах $H^{2}(\mathfrak{g} ; \mathfrak{g})$, если $p \neq 2$ (ср. [4] и [23]). 
Блок, Уилсон, Премет и Штраде, суммировав результаты тридцати лет работы нескольких групп исследователей и добавив новые идеи, доказали приведенную выше обобщенную КШ-гипотезу, см. [22]. При $p<5$ КШ-процедура и примеры Меликяна дают не все простые конечномерные алгебры Ли, появляются и другие примеры, а некоторые старые исчезают. В [13] мы вернулись к картановскому описанию $\mathbb{Z}$-градуированных алгебр Ли как алгебр Ли векторных полей, сохраняющих неинтегрируемые распределения, и, таким образом, проинтерпретировали «таинственные» исключительные примеры простых алгебр Ли при $p=3$ (Брауна, Франк, Ермолаева и Скрябина), пояснили кузнецовскую интерпретацию [16] алгебр Меликяна (как продолжений неотрицательной части исключительной алгебры Ли $\mathfrak{g}(2)$ в одной из ее $\mathbb{Z}$-градуировок) и обнаружили три новые серии простых алгебр Ли. В [1] тот же подход привел к открытию супералгебры Ли $\mathfrak{b j}$, простой супер-версии алгебры $\mathfrak{g}(2)$, и $\mathfrak{B j}(1 ; \underline{N} \mid 7)$, простой супер-версии алгебры Меликяна. Обе супералгебры - и $\mathfrak{b j}$, и $\mathfrak{B j}(1 ; \underline{N} \mid 7)$ - присущи лишь случаю $p=3$, а $\mathfrak{g}(2)_{\mathbb{K}}$ в этом случае не проста.

Мы напомним аналог КШ-гипотезы для $p>2$ (охватывающий и супералгебры Ли). Цель этой работы - привести аргументы в его пользу.

\section{§2. Классификация простых модулярных супералгебр Ли (гипотезы и результаты)}

В дополнение к упомянутым выше двум типам алгебр (алгебр вида $\mathfrak{g}(A)$ и алгебр векторных полей) в супер-случае есть еще один тип «симметричных» алгебр.

2.1. Странные супералгебры. Есть два супер-аналога алгебры Ли $\mathfrak{g l}(n)$ - непосредственный, $\mathfrak{g l}(m \mid n)$, и «странный», $\mathfrak{q}(n)$, сохраняющий комплексную структуру, заданную нечетным оператором, т. е. $\mathfrak{q}(n) \subset \mathfrak{g l}(n \mid n)$ состоит из всех операторов, сохраняющих нечетный оператор $J_{n \mid n}$, такой, что $J_{n \mid n}^{2}=-\mathrm{Id}$. У этой алгебры есть свой собственный (нечетный) странный след, выделяющий $\mathfrak{s q}(n)$. Супералгебры Ли $\mathfrak{p s q}(n)$ просты при $n>2$.

2.2. Исключительные супералгебры Ли. Недавно Эльдюк по-новому взглянул на магический квадрат Фрейденталя, и его подход (магический суперквадрат) открыл 10 новых примеров простых (исключительных) супералгебр Ли при $p=3$; еще двенадцать исключений вида $\mathfrak{g}(A)$ (см. предупреждение о «простоте» алгебр вида $\mathfrak{g}(A)$ в предыдущем параграфе) при $p=5,3$ и 2 были недавно получены вместе с полной классификацией в [5]. Описание супералгебр Эльдюка см. в [6], [8], [7], [9]; их описание в терминах матриц Картана и аналогов образующих Шевалле и соотношений Серра (и «не-Серра»), а также обозначения, которые мы используем ниже, см. в [2], [3], [5].

2.3. Супер-аналог КШ-гипотезы. Мы используем стандартные обозначения из [10], [22]; точные определения (алгоритм) КТЩ-продолжений (КартанаТанаки-Щепочкиной), в том числе частичных, см. в [21]. Пусть $m \mathfrak{g}(A)$ обозначает реализацию (супер)алгебры Ли $\mathfrak{g}(A)$ с помощью $m$-й матрицы Картана $A$, список и нумерацию которых см. в [13], [2], [3]. Градуировку $\operatorname{deg} e_{i}^{ \pm}= \pm s_{i}$ для образующих Шевалле $e_{i}^{ \pm}$(супер)алгебры Ли $\mathfrak{g}(A)$ назовем простейшей, если все координаты вектора $s=\left(s_{1}, \ldots, s_{n}\right)$ равны 0 и лишь одна «избранная» равна 1. Простая супералгебра ограничена, если ограничена ее четная часть; общее определение см. в [19]. 
В работе [19] сформулирован следующий аналог КШ-гипотезы, охватывающий и супералгебры. Он основан на совершенно иной идее, и в нем (как и в [13] и [1]) основную роль играют КТЩ-продолжения. (Именно так Картан получил все простые $\mathbb{Z}$-градуированные алгебры Ли полиномиального роста и конечной глубины, т. е. алгебры Ли типов (1), в то время, когда язык корней отсутствовал.)

Гипотеза. Над $\mathbb{K}$ у каждой простой конечномерной (супер)алгебрь Ли вида $\mathfrak{g}(A)$ или $\mathfrak{p s q}$ в одной из ее простейших $\mathbb{Z}$-градуировок возьмем неположительную часть и рассмотрим полные и частичные продолэсеня этой части, а в них - простые подфакторы, получаемые переходом $к$ производным алгебрам и факторизаиией по иентру. При $\underline{N}=(1, \ldots, 1)$ таким образом и методом КШ, примененным $\kappa$ супералгебрам Ли вида (1) над $\mathbb{C}$, получаются все ограниченные простые (супер)алгебры Ли при $p>2$.

При любом $\underline{N}$ и вместе с дебормаииями таким образом получаются все простые конечномерные модулярные (супер)алгебры Ли при $p>2$.

Наш основной результат - это найденные нами новые примеры простых модулярных супералгебр Ли $(\mathfrak{b r j}(2 ; 3), \mathfrak{b r j}(2 ; 5), \mathfrak{B} \mathfrak{R} \mathfrak{J}, \mathfrak{B j}(3 ; \underline{N} \mid 3), \mathfrak{B j}(3 \mid 5), \mathfrak{M e}(3 ; \underline{N} \mid 3))$, подкрепляющие нашу гипотезу.

2.4. Теорема Ямагучи [24]. Эта теорема, воспроизведенная в [13], [1], гласит, что для почти всех простых конечномерных алгебр Ли $\mathfrak{g}$ над $\mathbb{C} u$ ux

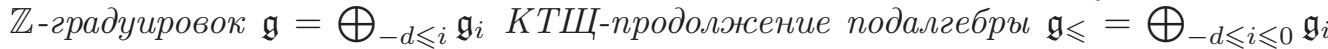
изоморфно алгебре Ли $\mathfrak{g}$, а редкими исключениями являются две из четырех серий простых бесконечномерных алгебр Ли векторных полей (еще две серии являются частичными продолжениями).

2.5. Аналоги теоремы Ямагучи. В следующих теоремах представлены результаты (полученные с помощью пакета программ SuperLie [11], но доступные непосредственной проверке) вычислений КТЩ-продолжений неположительных частей простых конечномерных (супер)алгебр Ли $\mathfrak{g}(A)$ с матрицей Картана $A$. Мы рассмотрели лишь простейшие $\mathbb{Z}$-градуировки, отвечающие каждой (а для бо́льших рангов - лишь избранным) из простейших.

Гипотеза. При $p>2$ другие $\mathbb{Z}$-градуировки рассмотренных нами (супер)алгебр Ли вида $\mathfrak{g}(A)$, так же, как и (супер)алгебр Ли $\mathfrak{g}$ бо́льших рангов (любого вида), не дадут новых простых (супер)алгебр Ли в качестве продолжений своих неположительных частей.

2.6. Теорема. В следующих случаях КТЩ-продолжение неположительной части алгебры Ли $\mathfrak{g}$ совпадает с $\mathfrak{g}$ : если $p=3$ и алгебра $\mathfrak{g}$ есть $\mathfrak{f}(4), \mathfrak{e}(6)$, $\mathfrak{e}(7)$ или $\mathfrak{e}(8)$, рассматриваемая $с \mathbb{Z}$-градуировкой, в которой «избран» лишь кокорень, отвечающий одной из крайних вершин на диаграмме Дынкина.

Гипотеза. (Доступным нам компьютерам не под силу проверить ее с помощью пакета SuperLie.) K случаям предъдущей теоремъ нужно добавить случай $p=5$ и алгебру $\mathfrak{g}=\mathfrak{e l}(5 ; 5)$ (см. [5]) в ее реализачии с единственным нечетным простым корнем и с $\mathbb{Z}$-градуировкой, в которой «избран» лишь один кокорень, отвечающий одной из крайних вериин на диаграмме Дынкина.

2.7. Теорема. Пусть $p=3$. Для всех известных ранее (мы нашли больше, см. теоремы в разд. 2.8 и 2.9) простых конечномерных супералгебр Ли $\mathfrak{g}$ ранга $\leqslant 3$ с матрищей Картана и для их простейших градуировок $s$ KТЩпродолжения (неположительной части алгебры $\mathfrak{g}$ ), отличные от $\mathfrak{g}$, даны в следующей таблище, поясненной в разд. 2.10-2.12. 


\begin{tabular}{|c|c|c|c|}
\hline $\mathfrak{g}$ & Матрица Картана & $s$ & имя продолженной алгебры \\
\hline $\mathfrak{o} \mathfrak{s p}(2 \mid 4)$ & $\left(\begin{array}{ccc}0 & 1 & 0 \\
-1 & 2 & -2 \\
0 & -1 & 2\end{array}\right)$ & $(010)$ & $\begin{cases}\mathfrak{o} \mathfrak{s p}(2 \mid 4) & \text { при } p>3, \\
\mathfrak{B j}(3 ; \underline{N} \mid 3) & \text { при } p=3\end{cases}$ \\
\hline $\begin{array}{c}\mathfrak{g}(2,3) \\
\text { см. }[2]\end{array}$ & $\left(\begin{array}{ccc}0 & 0 & -1 \\
0 & 0 & -2 \\
-1 & -2 & 2\end{array}\right)$ & $\begin{array}{c}(100) \\
(010)\end{array}$ & $\begin{array}{c}\mathfrak{B j}(2 \mid 4) \\
\mathfrak{B j}(3 \mid 5) \\
\mathfrak{b j}\end{array}$ \\
\hline
\end{tabular}

2.8. Супералгебры Меликяна для $\boldsymbol{p}=\mathbf{3}$. Известны две конструкции алгебр Меликяна $\mathfrak{M e}(5 ; \underline{N})=\bigoplus_{i \geqslant-2} \mathfrak{M e}(5 ; \underline{N})_{i}$, определенных при $p=5$ :

1) как КТЩ-продолжения тройки, состоящей из $\mathfrak{M e}_{0}=\mathfrak{c}(\mathfrak{v e c t}(1 ; \underline{1}))$, где $\mathfrak{c}(\mathfrak{g})$ - тривиальное центральное расширение (супер)алгебры Ли $\mathfrak{g}$ с одномерным центром, $\mathfrak{M e}_{-1}=\mathscr{O}(1 ; \underline{1}) /$ const и тривиального модуля $\mathfrak{M e}_{-2}$, см. [22]; эта конструкция была бы контрпримером к нашему аналогу КШ-гипотезы, если бы не конструкция, описанная в следующем пункте;

2) как полное КТЩ-продолжение неположительной части алгебры $\mathfrak{g}(2)$ в ее градуировке $s=(01)$, а $\mathfrak{g}(2)$ получается при этом как частичное продолжение, см. [16], [13].

В [1] мы выделили супералгебру Ли $\mathfrak{B j}(1 ; \underline{N \mid 7)}$ - простой аналог алгебры $\mathfrak{M e}(5 ; \underline{N})$ для $p=3$ - в виде частичного КТЩ-продолжения пары (отрицательная часть контактной супералгебры Ли $\left.\mathfrak{k}(1 ; \underline{N} \mid 7), \mathfrak{B j}(1 ; \underline{N} \mid 7)_{0}=\mathfrak{p g l}(3)\right)$, а супералгебру Ли $\mathfrak{b j}$ получили в качестве простого аналога алгебры $\mathfrak{g}(2)$ для $p=3$ с той же неположительной частью, что и у алгебры $\mathfrak{B j}(1 ; \underline{N} \mid 7)$. Таким образом, обе эти супералгебры Ли - и $\mathfrak{b j}$, и $\mathfrak{B j}(1 ; \underline{N} \mid 7)$ - получены нами в [2] с помощью супер-аналога конструкции 2).

Конструкция 1) Меликяна также имеет супер-аналог при $p=3$ (причем, как следует из классификации $\mathfrak{k}(2 n+1 \mid m)$-инвариантных дифференциальных операторов [20], только в ситуации, описанной в сформулированной ниже в этом разделе теореме), который приводит к новой серии простых супералгебр Ли в качестве полных продолжений, а также к другому простому аналогу алгебры $\mathfrak{g}(2)$ в качестве частичного продолжения; он же - супер-аналог алгебры Брауна $\mathfrak{b r}(2)$.

Теорема. При $p=3$ аналог конструкиии 1) Меликяна получается, если положить $\mathfrak{g}_{0}=\mathfrak{c}(\mathfrak{k}(1 ; \underline{1} \mid 1)), \mathfrak{g}_{-1}=\mathscr{O}(1 ; \underline{1} \mid 1) /$ const при тривиальном модуле $\mathfrak{g}_{-2}$. Мы обозначим полученную таким образом супералгебру Меликяна символом $\mathfrak{M e}(3 ; \underline{N} \mid 3)$; она не изоморфна супералгебре Меликяна $\mathfrak{B j}(1 ; \underline{N} \mid 7)$.

Обозначим символом brj $(2 ; 3)$ частичное КТЩ-продолжение с той же неположительной частью, что и у $\mathfrak{M e}(3 ; \underline{N} \mid 3)$. Это новая простая (исключительная) супералгебра Ли. У этой супералгебры три матрицы Картана: $\left(\begin{array}{cc}0 & -1 \\ 2 & 1\end{array}\right)$, $\left(\begin{array}{cc}0 & -1 \\ 1 & \overline{0}\end{array}\right) u\left(\begin{array}{cc}1 & -1 \\ -1 & \overline{0}\end{array}\right)$. Супералгебра Ли $\mathfrak{b r j}(2 ; 3)$ является супер-аналогом алгебры Брауна $\mathfrak{b r}(2)$, составляющей ее четную частъ: $\mathfrak{b r}(2)=\mathfrak{b} \mathfrak{r j}(2 ; 3)_{\overline{0}}$.

КТЩ-продолжения супералгебры Ли $1 \mathfrak{b r j}(2 ; 3)$ в их простейших градуировках приводят к известным простым супералгебрам Ли, тогда как частичные КТЩ-продолжения супералгебр Ли $2 \mathfrak{b r j}(2 ; 3)$ и $3 \mathfrak{b r j}(2 ; 3)$ в некоторых простейших градуировках дают новую простую супералгебру Ли, которую мы обозначаем через $\mathfrak{B R J}$. 
В отличие от алгебры Ли $\mathfrak{b r}(2)$ супералгебра Ли $\mathfrak{b r j}(2 ; 3)$ имеет аналог при $p=5$, а именно новую простую супералгебру Ли $\mathfrak{b r j}(2 ; 5)$, такую, что $\mathfrak{b} \mathfrak{r j}(2 ; 5)_{\overline{0}}=\mathfrak{s p}(4)$, с матрицами Картана $\left(\begin{array}{cc}0 & -1 \\ 2 & 1\end{array}\right)$ u $\left(\begin{array}{cc}0 & -1 \\ 3 & 2\end{array}\right)$. КТЩ-продолжсения супералгебры $\mathfrak{b r j}(2 ; 5)$ для всех ее матрии Картана и простейших градуировок s совпадают с самой супералгеброй $\mathfrak{b r j}(2 ; 5)$.

2.9. Простые супералгебры Ли $\mathfrak{g}(\boldsymbol{A})$. Когда мы обнаружили супералгебры Ли $\mathfrak{b} \mathfrak{j}$ и чуть не упустили реализацию $3 \mathfrak{b r j}(2 ; 3)$, мы решились классифицировать простые супералгебры вида $\mathfrak{g}(A)$. Вся классификация, в том числе при $p=2$, приведена в [5], а ниже - ее начало.

Теорема. При $p>5$ каждая простая конечномерная супералгебра Ли, не являющаяся алгеброй Ли, с матрищей Картана размера $2 \times 2$ изоморбна одной из следующих: osp $(1 \mid 4), \mathfrak{o s p}(3 \mid 2)$ или $\mathfrak{s l}(1 \mid 2)$. При $p=5$ ж этому списку следует добавить супералгебру Ли $\mathfrak{b r j}(2 ; 5)$, а при $p=3$ - супералгебру Ли $\mathfrak{b r j}(2 ; 3)$.

2.10. Описание супералгебры $\mathfrak{B j}(3 ; \underline{N} \mid 3)$. Вот реализация ее неположительной части:

\begin{tabular}{|l|l|}
\hline $\mathfrak{g}_{i}$ & образующие (четные $\mid$ нечетные) \\
\hline \hline $\mathfrak{g}_{-2}$ & $Y_{6}=\partial_{1} \mid Y_{8}=\partial_{4}$ \\
\hline \multirow{2}{*}{$\mathfrak{g}_{-1}$} & $Y_{2}=\partial_{2}, Y_{5}=x_{2} \partial_{1}+x_{5} \partial_{4}+\partial_{3} \mid$ \\
& $Y_{4}=\partial_{5}, Y_{7}=2 x_{2} \partial_{4}+\partial_{6}$ \\
\hline \multirow{2}{*}{$\simeq \mathfrak{g l}(1 \mid 1) \oplus \mathfrak{s l}(2) \oplus \mathbb{K}$} & $Y_{3}=x_{2}{ }^{2} \partial_{1}+x_{2} x_{5} \partial_{4}+x_{2} \partial_{3}+2 x_{5} \partial_{6}$, \\
& $Z_{3}=x_{3}^{2} \partial_{1}+2 x_{3} x_{6} \partial_{4}+x_{3} \partial_{2}+2 x_{6} \partial_{5}$, \\
& $H_{2}=2 x_{1} \partial_{1}+2 x_{2} \partial_{2}+x_{4} \partial_{4}+x_{5} \partial_{5}+2 x_{6} \partial_{6}$, \\
& $H_{1}=\left[Z_{1}, Y_{1}\right], H_{3}=\left[Z_{3}, Y_{3}\right] \mid$ \\
& $Y_{1}=x_{1} \partial_{4}+2 x_{2} \partial_{5}+x_{3} \partial_{6}$, \\
& $Z_{1}=2 x_{4} \partial_{1}+2 x_{5} \partial_{2}+x_{6} \partial_{3}$ \\
\hline
\end{tabular}

Здесь $\mathfrak{g}_{-1}$ - неприводимый над $\mathfrak{g}_{0}$ модуль с вектором старшего веса $Y_{2}$. В векторе $\underline{N}=\left(N_{1}, N_{2}, N_{3}\right)$ лишь $N_{1}$ может меняться, а $N_{2}=N_{3}=1$.

2.11. Описание супералгебры $\mathfrak{B j}(2 \mid 4)$. В этом и следующем разделах мы рассматриваем различные градуировки супералгебры Ли $\mathfrak{g}(2,3)$ с матрицей Картана, указанной в таблице из разд. 2.7. Эту супералгебру с $\mathbb{Z}$-градуировкой $s$ мы обозначим через $\mathfrak{g}(2,3 ; s)$. В этом разделе $s=(100)$ и $\operatorname{sdim}\left(\mathfrak{g}(2,3 ; s)_{-}\right)=2 \mid 4$. Поскольку $\mathfrak{g}(2,3 ; s)_{0}$-действие в модуле $\mathfrak{g}(2,3 ; s)_{-1}$ не точно, мы рассмотрим факторалгебру $\mathfrak{g}_{0}=\mathfrak{g}(2,3 ; s)_{0} / \mathfrak{a n n}\left(\mathfrak{g}_{-1}\right)$ и вложение $\left(\mathfrak{g}(2,3 ; s)_{-}, \mathfrak{g}_{0}\right) \subset \mathfrak{v e c t}(2 \mid 4)$. Вот эта реализация:

\begin{tabular}{|l|l|}
\hline $\mathfrak{g}_{i}$ & образующие (четные \\
\hline \hline $\mathfrak{g}_{-1}$ & $Y_{6}=\partial_{2}, Y_{8}=\partial_{1} \mid Y_{11}=\partial_{3}, Y_{10}=\partial_{4}, Y_{4}=\partial_{5}, Y_{1}=\partial_{6}$ \\
\hline \multirow{2}{*}{$\mathfrak{g}_{0} \simeq \mathfrak{o s p}(3 \mid 2)$} & $Y_{3}=x_{2} \partial_{1}+2 x_{4} \partial_{3}+x_{6} \partial_{5}, Y_{9}=\left[Y_{2},\left[Y_{3}, Y_{5}\right]\right]$, \\
& $Z_{3}=x_{1} \partial_{2}+2 x_{3} \partial_{4}+x_{5} \partial_{6}, Z_{9}=\left[Z_{2},\left[Z_{3}, Z_{5}\right]\right]$, \\
& $H_{2}=\left[Z_{2}, Y_{2}\right], H_{3}=\left[Z_{3}, Y_{3}\right] \mid$ \\
& $Y_{2}=x_{1} \partial_{4}+x_{5} \partial_{2}, Y_{5}=\left[Y_{2}, Y_{3}\right], Y_{7}=\left[Y_{3},\left[Y_{2}, Y_{3}\right]\right]$, \\
& $Z_{2}=x_{2} \partial_{5}+2 x_{4} \partial_{1}, Z_{5}=\left[Z_{2}, Z_{3}\right], Z_{7}=\left[Z_{3},\left[Z_{2}, Z_{3}\right]\right]$ \\
\hline
\end{tabular}


Здесь $\mathfrak{g}_{-1}-$ неприводимый над $\mathfrak{g}_{0}$ модуль с вектором старшего веса $Y_{1}$. КТЩпродолжение $\left(\mathfrak{g}_{-}, \mathfrak{g}_{0}\right)_{*}$ приводит к супералгебре Ли суперразмерности $13 \mid 14$. У этой супералгебры Ли есть три идеала $I_{1} \subset I_{2} \subset I_{3}$ с одной и той же неположительной частью, но разными положительными частями: $\operatorname{sdim}\left(I_{1}\right)=10 \mid 14$, $\operatorname{sdim}\left(I_{2}\right)=11\left|14, \operatorname{sdim}\left(I_{3}\right)=12\right| 14$. Идеал $I_{1}$ и есть наша супералгебра Ли $\mathfrak{b j}$, см. [1], [2].

2.12. Описание супералгебры $\mathfrak{B j}(\mathbf{3} \mid \mathbf{5})$. Здесь $s=(010)$ и $\operatorname{sdim}\left(\mathfrak{g}(2,3 ; s)_{-}\right)$ $=3 \mid 5$. Поскольку $\mathfrak{g}(2,3 ; s)_{0}$-действие в модуле $\mathfrak{g}(2,3 ; s)_{-1}$ не точно, мы рассматриваем факторалгебру $\mathfrak{g}_{0}=\mathfrak{g}(2,3 ; s)_{0} / \mathfrak{a n n}\left(\mathfrak{g}_{-1}\right)$ и вложение $\left(\mathfrak{g}(2,3 ; s)_{-}, \mathfrak{g}_{0}\right) \subset$ $\mathfrak{v e c t}(3 ; \underline{N} \mid 5)$ :

\begin{tabular}{|l|l|}
\hline $\mathfrak{g}_{i}$ & образующие (четные | нечетные) \\
\hline \hline $\mathfrak{g}_{-2}$ & $Y_{9}=\partial_{1} \mid Y_{10}=\partial_{3}, Y_{11}=\partial_{2}$ \\
\hline $\mathfrak{g}_{-1}$ & $Y_{8}=\partial_{4}, Y_{6}=\partial_{5} \mid Y_{5}=2 x_{4} \partial_{2}+2 x_{5} \partial_{3}+2 x_{7} \partial_{1}+\partial_{7}$, \\
& $Y_{2}=x_{4} \partial_{3}-2 x_{6} \partial_{1}+\partial_{8}, Y_{7}=x_{5} \partial_{2}+\partial_{6}$ \\
\hline $\mathfrak{g}_{0} \simeq \mathfrak{s l}(1 \mid 2)$ & $H_{1}=\left[Z_{1}, Y_{1}\right], H_{3}=\left[Z_{3}, Y_{3}\right]$ \\
& $Y_{3}=2 x_{3} \partial_{2}+2 x_{7} x_{8} \partial_{1}+x_{5} \partial_{4}+2 x_{7} \partial_{6}+x_{8} \partial_{7}$, \\
& $Z_{3}=2 x_{2} \partial_{3}+2 x_{6} x_{7} \partial_{1}+x_{4} \partial_{5}+x_{6} \partial_{7}+2 x_{7} \partial_{8} \mid$ \\
& $Y_{1}=2\left(2 x_{1} \partial_{3}+2 x_{6} x_{7} \partial_{2}+x_{6} \partial_{4}+x_{7} \partial_{5}\right)$, \\
& $Z_{1}=2\left(x_{3} \partial_{1}+2 x_{4} x_{5} \partial_{2}+2 x_{5} \partial_{3}+2 x_{5} x_{7} \partial_{1}+2 x_{4} \partial_{6}+x_{5} \partial_{7}\right)$, \\
& $Y_{4}=\left[Y_{1}, Y_{3}\right], Z_{4}=\left[Z_{1}, Z_{3}\right]$ \\
\hline
\end{tabular}

Здесь $\mathfrak{g}_{-1}-$ неприводимый над $\mathfrak{g}_{0}$ модуль с вектором старшего веса $Y_{2}$. Имеем $\operatorname{sdim}\left(\mathfrak{g}_{1}\right)=6 \mid 4$. У $\mathfrak{g}_{0}$-модуля $\mathfrak{g}_{1}$ есть два вектора младшего веса:

$$
\begin{array}{|l|l|}
\hline V_{1}^{\prime} & x_{1} x_{5} \partial_{2}+2 x_{5} x_{6} x_{8} \partial_{2}+x_{5} x_{7} x_{8} \partial_{3}+2 x_{1} \partial_{6}+2 x_{3} \partial_{4}+x_{5} x_{7} \partial_{4}+x_{5} x_{8} \partial_{5}+2 x_{7} x_{8} \partial_{7} \\
V_{1}^{\prime \prime} & x_{6} x_{7} x_{8} \partial_{2}+2 x_{1} \partial_{4}+x_{7} x_{8} \partial_{5} \\
\hline
\end{array}
$$

Оказывается, что $\mathfrak{g}_{0}$-модуль $\mathfrak{g}_{1}^{\prime \prime}$, порожденный векторами $V_{1}^{\prime}$ и $V_{1}^{\prime \prime}$, строго меньше чем $\mathfrak{g}_{1}$ и $\operatorname{sdim} \mathfrak{g}_{1}^{\prime \prime}=4 \mid 4$. Полное КТЩ-продолжение $\left(\mathfrak{g}_{-}, \mathfrak{g}_{0}, \mathfrak{g}_{1}\right)_{*}$ не просто, а вот частичное продолжение - подсупералгебра $\mathfrak{B j}(3 \mid 5):=\left(\mathfrak{g}_{-}, \mathfrak{g}_{0}, \mathfrak{g}_{1}^{\prime \prime}\right)_{*}$ проста. Отметим еще, что хотя $\mathfrak{B j}(3 \mid 5)$ и является подсупералгеброй Ли в $\mathfrak{v e c t}(3 ; \underline{N} \mid 5)$, сама она не зависит от $\underline{N}$. Поскольку ни у одной из известных простых конечномерных супералгебр Ли при $p=0$ или $p>3$ нет такой неположительной части ни в одной из $\mathbb{Z}$-градуировок, $\mathfrak{B j}(3 \mid 5)$ - новая (видимо, исключительная) супералгебра Ли.

\section{ЛитеРАтУРА}

[1] S. Bouarroudj, D. A. Leites, Simple Lie superalgebras and nonintegrable distributions in characteristic p [Простые супералгебры Ли и неинтегрируемые распределения в характеристике $p$ ], Зап. научн. сем. ПОМИ, 331 (2006), 15-29; http://arxiv.org/abs/math/0606682.

[2] S. Bouarroudj, P. Grozman, D. Leites, Cartan matrices and presentations of Elduque and Cunha simple Lie superalgebrasm, arXiv: http://arxiv.org/abs/math.RT/ 0611391.

[3] S. Bouarroudj, P. Grozman, D. Leites, Cartan matrices and presentations of the exceptional simple Elduque Lie superalgebra, http://arxiv.org/abs/math.RT/0611392. 
[4] S. Bouarroudj, P. Grozman, D. Leites, Infinitesimal deformations of symmetric simple modular Lie algebras and Lie superalgebras, http://arxiv.org/abs/0807.3054.

[5] S. Bouarroudj, P. Grozman, D. Leites, Classification of simple finite dimensional modular Lie superalgebras with Cartan matrix, http://arxiv.org/abs/0710.5149.

[6] I. Cunha, A. Elduque, An extended Freudenthal magic square in characteristic 3, http://arxiv.org/abs/math.RA/0605379.

[7] I. Cunha, A. Elduque, The extended Freudenthal Magic Square and Jordan algebras, http://arxiv.org/abs/math.RA/0608191.

[8] A. Elduque, New simple Lie superalgebras in characteristic 3, J. Algebra, 296:1 (2006), $196-233$.

[9] A. Elduque, Some new simple modular Lie superalgebras, http://arxiv.org/abs/ math.RA/0512654.

[10] W. Fulton, J. Harris, Representation theory. A first course, Graduate Texts in Math., vol. 129, Springer-Verlag, New York, 1991.

[11] P. Grozman, SuperLie, http://www.equaonline.com/math/SuperLie.

[12] P. Grozman, D. Leites, Defining relations for classical Lie superalgebras with Cartan matrix, Czech. J. Phys., 51:1 (2001), 1-22; http://arxiv.org/abs/hep-th/9702073.

[13] P. Grozman, D. Leites, Structures of $G(2)$ type and nonintegrable distributions in characteristic p, Lett. Math. Phys., 74:3 (2005), 229-262; http://arxiv.org/abs/ math.RT/0509400.

[14] В. Кац, Бесконечномерные алгебры Ли, Мир, М., 1993.

[15] А. И. Кострикин, И. Р. Шафаревич, Градуированные алгебры Ли конечной характеристики, Изв. АН СССР, сер. матем., 33:2 (1969), 251-322.

[16] M. I. Kuznetsov, The Melikyan algebras as Lie algebras of the type $G_{2}$, Comm. Algebra, 19:4 (1991), 1281-1312.

[17] A. Lebedev, Presentations of finite dimensional symmetric simple modular Lie algebras and superalgebras, PhD, Leipzig University, 2008.

[18] A. Lebedev, D. Leites (with Appendix by P. Deligne), On realizations of the Steenrod algebras, J. Prime Res. Math., 2:1 (2006), 101-112.

[19] D. Leites, Towards classification of simple finite dimensional modular Lie superalgebras in characteristic p, J. Prime Res. Math., 3 (2007), 101-110; MPIMiS preprint 132/2006 (www.mis.mpg.de).

[20] Д. Лейтес, Супералгебры Ли, в кн.: Итоги науки и техники. Современные проблемы математики. Новейшие достижения, т. 25, ВИНИТИ, М., 1984, 3-49.

[21] И. М. Щепочкина, Как реализовать алгебру Ли векторными полями, ТМФ, 147:3 (2006), 450-469; http://arxiv.org/abs/math.RT/0509472.

[22] H. Strade, Simple Lie algebras over fields of positive characteristic. I. Structure theory, de Gruyter Expositions in Math., vol. 38, Walter de Gruyter \& Co., Berlin, 2004.

[23] F. Viviani, Restricted simple Lie algebras and their infinitesimal deformations, http://arxiv.org/abs/math.RA/0702755.

[24] K. Yamaguchi, Differential systems associated with simple graded Lie algebras, in: Progress in differential geometry. Adv. Stud. Pure Math., vol. 22, Math. Soc. Japan, Tokyo, 1993, 413-494.

Department of Mathematics, United Arab Emirates University e-mail: Bouarroudj.sofiane@uaeu.ac.ae

Поступило в редакцию

Equa Simulation AB, Stockholm

e-mule: pavel@rixtele.com

Department of Mathematics, University of Stockholm

e-mail:mleites@math.su.se

9 декабря 2006 г. 